\title{
Ginkgo Biloba Extract EGB761 Protects against Aging-Associated Diastolic Dysfunction in Cardiomyocytes of D-Galactose-Induced Aging Rat
}

\author{
Jing Liu, ${ }^{1}$ Junhong Wang, ${ }^{1}$ Xiangjian Chen, ${ }^{2}$ Changqing Guo, ${ }^{3}$ Yan Guo, ${ }^{1}$ and Hui Wang ${ }^{4}$ \\ ${ }^{1}$ Department of Gerontology, The First Affiliated Hospital of Nanjing Medical University, Nanjing, Jiangsu 210029, China \\ ${ }^{2}$ Institute of Cardiovascular Disease, The First Affiliated Hospital of Nanjing Medical University, Nanjing, Jiangsu 210029, China \\ ${ }^{3}$ Department of Cardiology, Anyang Sixth People Hospital, Anyang, Henan 455000, China \\ ${ }^{4}$ Department of Cardiology, The Shengze Hospital of Jiangsu Province, SuZhou 215002, China
}

Correspondence should be addressed to Yan Guo, guoyan51@hotmail.com and Hui Wang, wangnuo@263.net

Received 30 November 2011; Revised 6 March 2012; Accepted 13 March 2012

Academic Editor: Ana Fortuno

Copyright (๑) 2012 Jing Liu et al. This is an open access article distributed under the Creative Commons Attribution License, which permits unrestricted use, distribution, and reproduction in any medium, provided the original work is properly cited.

\begin{abstract}
The aim of the present study was to make use of the artificially induced aging model cardiomyocytes to further investigate potential anti-aging-associated cellular diastolic dysfunction effects of EGB761 and explore underlying molecular mechanisms. Cultured rat primary cardiomyocytes were treated with either D-galactose or D-galactose combined with EGB761 for $48 \mathrm{~h}$. After treatment, the percentage of cells positive for SA- $\beta$-gal, AGEs production, cardiac sarcoplasmic reticulum calcium pump (SERCA) activity, the myocardial sarcoplasmic reticulum calcium uptake, and relative protein levels were measured. Our results demonstrated that in vitro stimulation with D-galactose induced AGEs production. The addition of EGB761 significantly decreased the number of cells positive for SA- $\beta$-gal. Furthermore, decreased diastolic $\left[\mathrm{Ca}^{2+}\right]_{i}$, curtailment of the time from the maximum concentration of $\mathrm{Ca}^{2+}$ to the baseline level and increased reuptake of $\mathrm{Ca}^{2+}$ stores in the SR were also observed. In addition, the level of pSer16-PLN protein as well as SERCA was markedly increased. The study indicated that EGb761 alleviates formation of AGEs products on SERCA2a in order to mitigate myocardial stiffness on one hand; on other hand, improve SERCA2a function through increase the amount of Ser16 sites PLN phosphorylation, which two hands finally led to ameliorate diastolic dysfunction of aging cardiomyocytes.
\end{abstract}

\section{Introduction}

Diastolic heart failure (DHF) is most commonly seen in elderly patients and women with a history of diabetes, hypertension, obesity, cardiac ischemia, and so on. Existing research revealed that elevated oxidative stress, $\mathrm{NAD}(\mathrm{P}) \mathrm{H}$ oxidase expression, protein carbonyl formation, protein oxidation, and protein modification as well as enhanced AGE level have been found in aged cardiac myocytes [1]. The major cause for DHF is cardiac aging, which is referred to as a dramatic decline in cardiac pump function with advanced age and resulted in diastolic dysfunction [2, 3]. Due to lack of understanding the mechanism of cardiac diastolic dysfunction, current clinical treatment for patients with DHF is disappointing [4-6]. Therefore, understanding mechanism of aging myocardial diastolic dysfunction in molecular level is especially important for the specific therapeutic targets of DHF association with aging.

$\mathrm{D}$-galactose is a reducing sugar that reacts readily with the free amines of amino acids in proteins and peptides both in vitro and in vivo to form advanced glycation endproducts (AGEs) [4]. AGE is increased during aging and has been linked to the pathogenesis of many ageassociated pathologies such as diabetes, arteriosclerosis, nephropathy, infection, and Alzheimer's disease [5]. Our previous study demonstrated that D-galactose injection led to accelerated aging phenotypes manifested by an increased AGEs level, and aging rats fed with D-galactose present 
significant cardiac remodeling compared with adult rats, which was manifested by increased left ventricular posterior wall and interventricular septal thickness and by increased left ventricular weight and impaired diastolic function. The contents of MDA, mtDNA, and AGEs were significantly increased; however, the contents of SOD and GSH-PX were decreased. Therefore, we have simulated aging process in vivo by $\mathrm{D}$-galactose intervention in order to study aging-related cardiac diastolic dysfunction.

The incidence of cardiac diastolic dysfunction is related to an elevated myocardial stiffness and the resting tension $\left(F_{\text {passive }}\right)$ which led to diastolic capacity decline. The increase in myocardial stiffness is mainly related to AGEs crosslinking with collagen deposition on myocardial interstitium, whereas the latter is mainly related to abnormal myocardial sarcoplasmic reticulum calcium transport regulatory proteins which led the intracellular calcium overload in diastolic phase [6]. Dysregulation of intracellular calcium homeostasis plays a significant role in the aged myocardial cell, which leads to diastolic heart failure. An excessive increase in the intracellular concentration of $\mathrm{Ca}^{2+}$ and impaired translocating calcium ions from the cytosol to the lumen of the sarcoplasmic reticulum would result in $\mathrm{Ca}^{2+}$ overload and thus produce myocardial diastolic dysfunction [2].

As intracellular calcium is mainly regulated by the activity of myocardial sarcoplasmic reticulum calcium transport regulatory proteins, removal of calcium from the cytosol may be delayed by a decrease in the activity of sarco/endoplasmic reticulum calcium adenosine triphosphate (SERCA) or an increase in the level of activity of phospholamban (PLN), which is a SERCA-inhibitory protein [7]. SERCA and PLN dysfunction may lead to intracellular calcium overload, which further arouse diastolic dysfunction [8].

EGb761 is a standard extract from the leaves of Ginkgo biloba (Yinxing) containing 24\% ginkgo-flavone glycosides (e.g., kaempferol, quercetin, and isorhamnetin derivatives) and $6 \%$ terpenoid (e.g., ginkgolides A, B, C, J and bilobalide) [9]. Numerous studies have shown that the flavonoid components scavenge superoxide, hydroxyl radicals, and nitric oxide (NO) and protect myocardia from ischemiareperfusion injury [10-13], and the terpenoid constituents also showed their cardioprotective effects independent from the free radical-scavenging properties [14].

The cardioprotective effects of EGb761 have been demonstrated in various in vivo and in vitro animal models and humans. The existing literature $[12,15]$, including our prophase research, has highlighted that EGb761 has particular antiaging effects on cardiomyocytes by inhibiting nonenzymatic glycation and reducing the deposition of AGEs in myocardium tissues $[12,16]$. However, due to cardiac aging which is referred to diastolic dysfunction, it is unclear yet whether EGb761 can regulate myocardial sarcoplasmic reticulum calcium transport regulatory proteins to improve the diastolic function of aging cardiomyocytes. In this study, we then aimed to investigate the possible action and its molecular mechanisms in cultured D-galactoseinduced aging rat cardiomyocytes of treatment EGB761.

\section{Materials and Methods}

2.1. Drugs and Reagents. Standard EGb761 preparation that we used mainly contains $4.2 \mathrm{mg}$ flavonoid from Dr. Willmar Schwabe Pharmaceuticals (Karlsruhe, Germany); D-galactose and caffeine were purchased from Sigma Aldrich Inc (G5388; C1778); Dulbecco's modified Eagle's medium (DMEM) and fetal calf serum (FCS) were purchased from Gibco Invitrogen (31600-034; 16000044); senescence $\beta$ galactosidase $(\beta$-gal) staining kit was purchase from Cell Signaling (no. 9860); rat AGEs ELISA kit was purchased from ADL Technology Inc (E0263r). The enhanced chemiluminescence (ECL) Western blotting detection kit was purchased from Pierce Rockford, IL, USA. Other materials used are specified in detail in the following sections.

2.2. Primary Culture of Cardiomyocytes. Neonatal cardiomyocytes were obtained from 20 Sprague-Dawley rats aged 1-2 days. Cardiac tissue was harvested using previously described protocols with minor modifications [17]. The ventricles from the rats were minced and dissociated in $0.06 \%$ trypsin at $37^{\circ} \mathrm{C}$. The dissociated myocytes were placed into DMEM supplemented with $20 \%$ fetal calf serum and $100 \mathrm{U} / \mathrm{mL}$ penicillin and streptomycin in a $50 \mathrm{~mL}$ Corning cell culture flask for $1.5 \mathrm{~h}$ at $37^{\circ} \mathrm{C}$ with $5 \% \mathrm{CO}_{2}$, which minimized nonmyocyte cells by adhesion to the bottom of the flask. The nonattached myocytes were then removed and plated $\left(5-6 \times 10^{5} / \mathrm{mL}\right)$ into a six-well culture cluster at $37^{\circ} \mathrm{C}$ in room air with $5 \% \mathrm{CO}_{2}$. After incubation for $48 \mathrm{~h}, 80 \%$ of the attached myocytes were beating spontaneously and were used for further experiments. All procedures were performed in accordance with the Guide for the Care and Use of Laboratory Animals (National Institutes of Health Publication no. 85-23, revised 1996).

2.3. D-Galactose Treatment Protocol. The treatment protocol for D-galactose induction was as previously described [18]. Once the attached myocytes were beating spontaneously, the DMEM supplemented with $20 \%$ fetal calf serum was removed, and DMEM supplemented with $5 \mathrm{~g} / \mathrm{L}$ D-galactose was added to the cardiomyocytes in the culture cluster for a further $48 \mathrm{~h}$ incubation period. In addition, for the cardioprotection study, EGB761 plus D-galactose was added into the culture media at concentrations of 5, 10, and $20 \mu \mathrm{g} \cdot \mathrm{mL}^{-1}$ throughout the treatment.

2.4. SA $\beta$-Gal Staining. Senescence-associated $\beta$-gal (SA- $\beta$ gal) activity was measured with the $\beta$-gal staining kit at $\mathrm{pH}$ 6.0 according to the instructions from the manufacturer. Briefly, cells were washed in phosphate buffered saline (PBS), fixed for $10-15 \mathrm{~min}$ at room temperature with $1 \mathrm{~mL}$ of fixative solution and incubated overnight at $37^{\circ} \mathrm{C}$ with the staining solution mix. Cells were observed for development of the blue coloration with a microscope at a magnification of $\times 400$.

2.5. AGEs ELISA Assay. The AGEs assay was performed with AGEs ELISA kit according to the instructions from the 
manufacturer. The reagents of the test kit were placed at room temperature for $30 \mathrm{~min}$ and diluted $1: 20$ with distilled water. Aliquots of $100 \mu \mathrm{L}$ of the standards and samples were added to blank micropores and $50 \mu \mathrm{L}$ enzyme marker solution was added. Microtiter plates were incubated at $37^{\circ} \mathrm{C}$ for $60 \mathrm{~min}$ and then washed five times and put aside for 10$20 \mathrm{~s}$ each time. The A and B substrate solutions $(50 \mu \mathrm{L})$ were added into the microtiter plates for $15 \mathrm{~min}$ dark reactions at $37^{\circ} \mathrm{C}$. The reaction was terminated by the addition of $50 \mu \mathrm{L}$ stop solution, and the optical density (OD) at $450 \mathrm{~nm}$ was determined by an ultra microplate reader (ELX 808 IU, Bio-Tek Instruments Inc.). An AGEs standard curve was generated and the AGEs values of the samples were calculated from the standard curve.

2.6. Measurement of Cardiac SERCA Activity. SERCA activity was determined by a modified p-nitrophenyl phosphate (p-NPP) method routinely used in our laboratory [19]. Briefly, cardiomyocytes were homogenized and centrifuged at $4^{\circ} \mathrm{C}$ for $15 \mathrm{~min}$. A $10 \mu \mathrm{L}$ aliquot of supernatant was preincubated for $10 \mathrm{~min}$ at $37^{\circ} \mathrm{C}$ with $80 \mu \mathrm{L}$ buffer solution containing: $10 \mu \mathrm{L} \mathrm{MgCl}_{2}, 10 \mu \mathrm{L}$ EGTA, $10 \mu \mathrm{L} \mathrm{KCl,} 30$ or $40 \mu \mathrm{L}$ HEPES ( $\mathrm{pH} 7.4$ ), and $10 \mu \mathrm{L} 0.01 \%$ TritonX-100, either with or without $10 \mu \mathrm{L} \mathrm{CaCl}_{2}$. The phosphatase reaction was started by the addition of $10 \mu \mathrm{L} 10 \mathrm{mM}$ p-NPP disodium salt hexahydrate and stopped after $30 \mathrm{~min}$ by the addition of $100 \mu \mathrm{L} 500 \mathrm{mM}$ Tris (pH 8.7) and $55 \mathrm{mM}$ EDTA. The liberated p-nitrophenol was measured by an ultra microplate reader (ELX $808 \mathrm{IU}$ ) at a wavelength of $405 \mathrm{~nm}$ and a standard curve was generated. The SERCA activity was expressed as the amount of $\mathrm{p}$-nitrophenol produced per gram of protein in a minute $(\mu \mathrm{mol} / \mathrm{g} / \mathrm{min})$.

2.7. Measurement of $\left[\mathrm{Ca}^{2+}\right]_{i}$ and $\mathrm{SR} \mathrm{Ca}^{2+}$ Load. Field myocyte on the cell culture dishes (Corning Inc) and $\left[\mathrm{Ca}^{2+}\right]_{i}$ were measured as previously described [19], using Fura2/AM $\left(2.0 \mu \mathrm{M}\right.$, Invitrogen) for $30 \mathrm{~min}$ at $37^{\circ} \mathrm{C}$. SR $\mathrm{Ca}^{2+}$ load was measured as the $\Delta\left[\mathrm{Ca}^{2+}\right]_{i}$ amplitude upon rapid exposure to $10 \mathrm{mM}$ caffeine. The ratio of backgroundcorrected fluorescence at $340 \mathrm{~nm}$ excitation to $380 \mathrm{~nm}$ excitation (F340/F380 ratio) was regarded as $\left[\mathrm{Ca}^{2+}\right]_{i}$. The time from the maximum concentration of $\mathrm{Ca}^{2+}$ to the baseline level was regarded as the time of SR $\mathrm{Ca}^{2+}$ uptake.

2.8. Western Blot. Cells were lysed with ice-cold Cell Lysis Reagent containing protease and phosphatase inhibitors ( $\mathrm{Bi}$ Yun Tian, China, P0013) to extract cytoplasm proteins. The protein concentrations of the cytoplasm were determined by bicinchoninic acid (BCA) protein concentration determination. Equal amounts of protein extracts were separated to $16 \%$ SDS/PAGE and blotted onto a polyvinylidene difluoride (PVDF) membranes (Millipore, Technology Inc). The membrane was blocked and probed overnight at $4^{\circ} \mathrm{C}$ with primary antibodies against SERCA2a (1:1000, ARB Inc.) and Ser16/Thr17-phosphorylated phospholamban (pPLN) (1:1000, Abcam Inc/ $1: 100$, Santa Cruz Inc) and GAPDH (1:3000, Sigma), followed by incubation with horseradish-peroxidase-conjugated secondary antibody for
$1 \mathrm{~h}$ at room temperature. Blots were developed using an ECL detection system and exposed to X-ray film. Normalized bands densities were analyzed by Gel-pro analyzer software and expressed as ratios to GAPDH.

2.9. Statistical Analysis. All data are presented as mean \pm standard deviation (SD). Statistical analysis was performed by ANOVA for multiple comparisons. Origin 7.0 (OriginLab, USA) was used to perform all statistical analysis. In each case, $P<0.05$ was considered statistically significant.

\section{Results}

3.1. EGB761 Prevented against Increased Numbers of $S A$ $\beta$-Gal-Positive Cells and Formation of AGEs. To investigate whether cardiomyocytes-treated D-galactose showed a senescent phenotype, we examined the numbers of SA $\beta$ gal-positive cells in all groups of cardiomyocytes. In Dgalactose-induced cardiomyocytes, the ratio of SA $\beta$-galpositive cardiomyocytes was significantly increased than that of control cardiomyocytes. The addition of EGB761 significantly decreased the number of cells positive for SA- $\beta$-gal in a dose-dependent manner, with an optimal concentration of $20 \mu \mathrm{g} \cdot \mathrm{mL}^{-1}(n=20$ number of rats each group and $n=6$ experimental replicates; $P<0.05$ versus $\mathrm{D}$-galactose).

Meanwhile, AGEs are considered as one of the important markers in cardiac aging. In the present study, AGEs content was significantly increased in the D-galactose-treated group. Significantly decreased AGEs level was observed in different concentrations of EGB761 treatment groups ( $n=20$ number of rats each group and $n=6$ experimental replicates; $P<$ 0.05 versus D-galactose) (Table 1 and Figure 1 ).

3.2. The Effect of EGB761 on the Intracellular $\left[\mathrm{Ca}^{2+}\right]_{i}$ and $\mathrm{SR} \mathrm{Ca}^{2+}$ Load. Intracellular $\mathrm{Ca}^{2+}$ overload is suggested to be one of the main factors involved in DHF. We then used the $\mathrm{Ca}^{2+}$ indicator Fluo2/AM to determine the effect of AGEs on the intracellular $\left[\mathrm{Ca}^{2+}\right]_{i}$, the time that $\mathrm{Ca}^{2+}$ uptake into sarcoplasmic reticulum and $\mathrm{SR} \mathrm{Ca}^{2+}$ load. In the D-galactose group, an increased diastolic $\left[\mathrm{Ca}^{2+}\right]_{i}$ was observed, and the time from the maximum concentration of $\mathrm{Ca}^{2+}$ to the baseline level was prolonged compared with the controls $(P<0.05)$. After perfusion with $10 \mathrm{mM}$ caffeine, the amplitude of the caffeine-induced calcium transient $\left(\Delta\left[\mathrm{Ca}^{2+}\right]_{i}\right)$ also declined in D-galactose-treated group. However, above-mentioned effects were reversed by $20 \mu \mathrm{g} \cdot \mathrm{mL}^{-1}$ EGB761 ( $n=20$ number of rats each group and $n=6$ experimental replicates; $P<0.05$ versus $D$-galactose). Representative changes in the ratio are shown in Table 2.

3.3. The Effect of EGB761 on SERCA Activity in Cardiomyocytes. To investigate the mechanism of EGB761 on intracellular $\left[\mathrm{Ca}^{2+}\right]_{i}$ and SR $\mathrm{Ca}^{2+}$ load., the SR $\mathrm{Ca}^{2+}$-ATPase (SERCA) activity in the cardiomyocytes was measured (Table 3). An obvious suppression of SERCA activity was observed in the D-galactose group, and this inhibition effect was attenuated by $20 \mu \mathrm{g} \cdot \mathrm{mL}^{-1}$ EGB761 treatment $(n=20$ 
TABLE 1: Effects of EGB761 on the numbers of SA $\beta$-gal-positive cell and formation of AGEs.

\begin{tabular}{lccccc}
\hline Group & $\begin{array}{c}\text { Control } \\
(N=20)\end{array}$ & $\begin{array}{c}5 \mathrm{~g} \cdot \mathrm{L}^{-1} \\
\text {-galactose } \\
(N=20)\end{array}$ & $\begin{array}{c}5 \mathrm{~g} \cdot \mathrm{L}^{-1} \mathrm{D} \text {-galactose }+ \\
5 \mu \mathrm{g} \cdot \mathrm{mL}^{-1} \mathrm{EGB761} \\
(N=20)\end{array}$ & $\begin{array}{c}5 \mathrm{~g} \cdot \mathrm{L}^{-1} \mathrm{D} \text {-galactose }+ \\
10 \mu \mathrm{g} \cdot \mathrm{mL}^{-1} \mathrm{EGB761} \\
(N=20)\end{array}$ & $\begin{array}{c}5 \mathrm{~g} \cdot \mathrm{L}^{-1} \mathrm{D} \text {-galactose }+ \\
20 \mu \mathrm{g} \cdot \mathrm{mL} \mathrm{L}^{-1} \mathrm{EGB761} \\
(N=20)\end{array}$ \\
\hline $\begin{array}{l}\text { The percentage of SA } \\
\beta \text {-gal-positive cell }(\%)\end{array}$ & $17.15 \pm 2.9$ & $75.6 \pm 4.9^{\mathrm{a}}$ & $57.7 \pm 7.9^{\mathrm{b}}$ & $49.7 \pm 9.2^{\mathrm{c}}$ & $34.0 \pm 6.6^{\mathrm{d}}$ \\
AGEs content $(\mathrm{pg} / \mathrm{mL})$ & $93.22 \pm 26.14$ & $702.58 \pm 32.16^{\mathrm{a}}$ & $404.36 \pm 32.94^{\mathrm{b}}$ & $356.77 \pm 25.32^{\mathrm{c}}$ & $248.72 \pm 77.26^{\mathrm{d}}$ \\
\hline
\end{tabular}

(Data are means $\pm \mathrm{SD}, n=6$ experimental replicates, ${ }^{\mathrm{a}} P<0.05$ Versus control group; ${ }^{\mathrm{b}} P<0.05,{ }^{\mathrm{c}} P,{ }^{\mathrm{d}} P<0.05$ Versus $\mathrm{D}$-galactose group).

TABLE 2: Effects of EGB761 on intracellular diastolic $\left[\mathrm{Ca}^{2+}\right]_{i}$, the time from the maximum concentration of $\mathrm{Ca}^{2+}$ to the baseline level and SR $\mathrm{Ca}^{2+} \operatorname{load}\left(\Delta\left[\mathrm{Ca}^{2+}\right]_{i}(\%)\right)$.

\begin{tabular}{|c|c|c|c|}
\hline Group & $\begin{array}{l}\text { Control } \\
(N=20)\end{array}$ & $\begin{array}{c}5 \mathrm{~g} \cdot \mathrm{L}^{-1} \mathrm{D} \text {-galactose } \\
\quad(N=20)\end{array}$ & $\begin{array}{c}5 \mathrm{~g} \cdot \mathrm{L}^{-1} \mathrm{D} \text {-galactose }+20 \mu \mathrm{g} \cdot \mathrm{mL}^{-1} \text { EGB761 } \\
(N=20)\end{array}$ \\
\hline$\left(\left[\mathrm{Ca}^{2+}\right]_{i}\right)_{\mathrm{D}}$ & $0.59 \pm 0.06$ & $0.71 \pm 0.08^{\mathrm{a}}$ & $0.61 \pm 0.07^{\mathrm{b}}$ \\
\hline$t_{\beta(\mathrm{ms})}$ & $775.15 \pm 121.68$ & $1000.95 \pm 129.34^{\mathrm{a}}$ & $834.36 \pm 301.85^{\mathrm{d}}$ \\
\hline${ }_{\Delta}\left[\mathrm{Ca}^{2+}\right]_{i}(\%)$ & $0.53 \pm 0.19$ & $0.11 \pm 0.02^{\mathrm{a}}$ & $0.47 \pm 0.16^{\mathrm{b}}$ \\
\hline
\end{tabular}

(Data are means $\pm \mathrm{SD}, n=6$ experimental replicates, ${ }^{\mathrm{a}} P<0.05$ versus control group; ${ }^{\mathrm{b}} P<0.05$ versus $\mathrm{D}$-galactose group).

TABLE 3: Effects of EGB761 on sarcoplasmic reticulum calcium pump (SERCA2a) activity.

\begin{tabular}{lc}
\hline Group & $\begin{array}{c}\text { SERCA2a activity } \\
(\mu \mathrm{mol} / \mathrm{g} / \mathrm{min})\end{array}$ \\
\hline Control & $2.68 \pm 0.35$ \\
$5 \mathrm{~g} \cdot \mathrm{L}^{-1} \mathrm{D}$-galactose & $1.60 \pm 0.28^{\mathrm{a}}$ \\
$5 \mathrm{~g} \cdot \mathrm{L}^{-1} \mathrm{D}$-galactose $+20 \mu \mathrm{g} \cdot \mathrm{mL}^{-1} \mathrm{EGB761}$ & $2.43 \pm 0.44^{\mathrm{b}}$ \\
\hline
\end{tabular}

(Data are means $\pm \mathrm{SD}, n=6$ experimental replicates, ${ }^{\text {a }} P<0.05$ versus control group; ${ }^{\mathrm{b}} P<0.05$ versus $\mathrm{D}$-galactose group).

number of rats each group and $n=6$ experimental replicates; $P<0.05$ versus D-galactose).

\subsection{The Effect of EGB761 on Sarcoplasmic Reticulum Calcium} Transport Regulatory Proteins Expression in Cardiomyocytes. In order to investigate the underlying mechanisms of EGB761 protecting against aging-associated diastolic dysfunction in cardiomyocytes, protein levels of calcium transport regulatory proteins were measured by Western blot. As shown in Figure 2, an approximately 60\% downregulation of SERCA2a protein expression was observed in D-galactosetreated cardiomyocytes, while it was increased in addition of EGB761 compared with the D-galactose group $(P<$ $0.05)$. Since the inhibitory effect of PLN on SERCA2a was dependent on the phosphorylation level of PLN, pSer ${ }^{16}$-PLN and $\mathrm{p}$-Thr ${ }^{17}$-PLN protein expressions were then measured. As shown in Figure 2, the level of p-Ser ${ }^{16}$-PLN protein expression was markedly decreased by $54 \%$ in the Dgalactose-treated cardiomyocytes compared with the control group, while it was increased in the EGB761 group $(P<$ 0.05 versus $\mathrm{D}$-galactose). However, the level of $\mathrm{p}$-Thr ${ }^{17}$-PLN protein expression showed no significant changes in any groups $(n=20$ number of rats and $n=6$ experimental replicates; $P<0.05$ versus $D$-galactose).

\section{Discussion}

With the discovery of in vivo derived AGEs and then several cell-surface AGE-binding proteins, much research has been devoted to studying AGE formation and the potential biological effects of AGEs [20]. Several earlier reports showed that free radicals were increased in D-galactose-treated animals and suggested that the increased free radicals may account for the underlining mechanism responsible for the acceleration of aging [21, 22]. However, data in earlier study from Song et al. has shown that the aging change is not D-galactose specific and aminoguanidine as an AGE inhibitor preventing sugar-induced aging changes suggests that glycation, rather than free radicals, is the major cause of aging in this model [4]. In our current study, myocardial cells chronical incubation with D-galactose has been used as an artificially induced cardiomyocytes aging model for AGEs formation and to further investigate potential antiaging-associated diastolic dysfunction effects of EGB761 and underlying molecular mechanisms.

The senescent phenotypes are associated with a typical gene expression profile [23]; a senescent morphology increases in senescence-associated $\beta$-galactosidase (SA $\beta$ gal) activity [24]. Our data showed that the numbers of SA $\beta$-gal-positive cells were significantly increased in all of cardiomyocytes after D-galactose treatment for $48 \mathrm{~h}$. Meanwhile, intracellular AGEs content was significantly elevated compared with control group. It is confirmed that D-galactose-induced cardiomyocytes aging model was successfully established in vitro. It is in agreement with those ideas in the previous literature that is accumulation of AGEs may associate with aging [1]. When EGB761 was included, however, the numbers of SA $\beta$-gal-positive cells and intracellular AGEs content were significantly decreased, which may indicate that EGB761 can attenuate the intracellular formation of AGEs and delay the cellular senescence.

Relaxation dysfunction has been indicated in the aging myocardium [25]. At the cellular level, depressed relaxation 


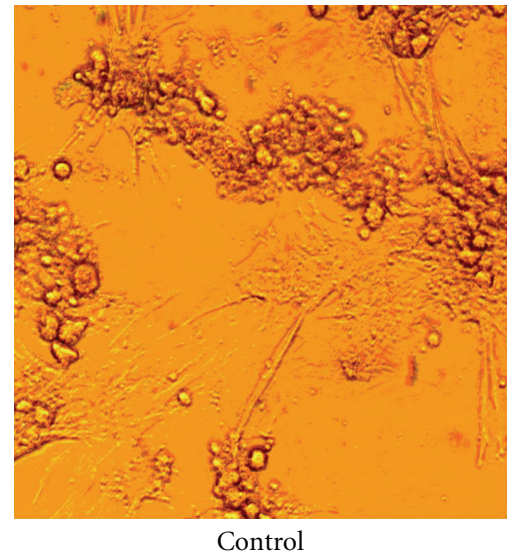

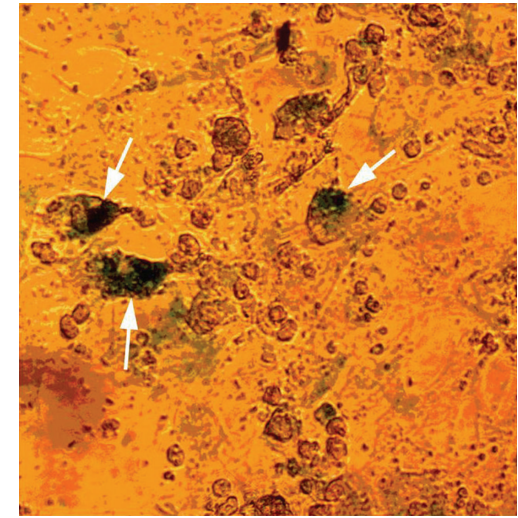

D-galactose

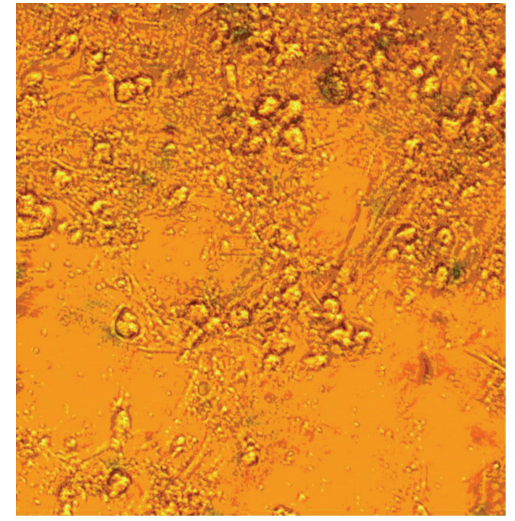

D-galactose + EGB761

$(\times 400)$

Figure 1: Response of neonatal rat cardiomyocytes to $5 \mathrm{~g} \cdot \mathrm{L}^{-1} \mathrm{D}$-galactose and $5 \mathrm{~g} \cdot \mathrm{L}^{-1} \mathrm{D}$-galactose combined with $20 \mu \mathrm{g} \cdot \mathrm{mL}-1 \mathrm{EGB} 761$ for $48 \mathrm{~h}$. Phase-contrast images showing morphologic changes and stained cells. (SA- $\beta$-gal positive cells; Blue, magnification $\times 400)$.

reflects impaired removal of cytosolic $\mathrm{Ca}^{2+}$ and reduced cardiac SR $\mathrm{Ca}^{2+}$ loading. Data from our present study depicted that AGEs accumulated on cardiomyocytes lead to impaired intracellular $\mathrm{Ca}^{2+}$ handling, which is the main cause of increased diastolic $\left[\mathrm{Ca}^{2+}\right]_{i}$ and a prolonged relaxation duration in aged cardiomyocytes. Meanwhile, similar with the results previous reported by Petrova et al. [26]. As early as 1996, it was reported that all of these $\mathrm{Ca}^{2+}$ removal systems are functionally inhibited in diabetes [27]. Our results also demonstrated that caffeine-induced $\mathrm{Ca}^{2+}$ transients, an indication of the reserve of $\mathrm{Ca}^{2+}$ in the SR, were significantly reduced. However, given EGB761 could increase reuptake of $\mathrm{Ca}^{2+}$ stores in the SR, curtailment of the time of SR $\mathrm{Ca}^{2+}$ uptake and a decrease in the diastolic $\left[\mathrm{Ca}^{2+}\right]_{i}$ in cardiomyocytes. These findings have suggested that EGB761 may protect against aging-associated diastolic dysfunction in cardiomyocytes.

Simultaneously $\mathrm{Ca}^{2+}$ cycling is a critical determinant of cardiomyocyte contractility and dilation. In mammalian, during relaxation, about $70 \%$ of cytosolic $\mathrm{Ca}^{2+}$ is partly taken back into the sarcoplasmic reticulum by SERCA2a under the regulation of PLN and partly extruded to the external medium through the action of plasma membrane $\mathrm{Ca}^{2+}$ ATPase (PMCA,2\%) and the $\mathrm{Na}^{+} / \mathrm{Ca}^{2+}$ exchanger (NCX, $28 \%$ ) [28]. Thus, changed level and/or activity of SERCA2a protein and/or its regulatory protein PLN may influence the homeostasis of cardiac intracellular $\mathrm{Ca}^{2+}$ [29]. As the PLN phosphorylation status is the key factor regulating the function of SERCA, the main phosphorylation status of Ser16 and Thr17 sites of PLN was investigated in our study [30]. Our data revealed a significant suppression of SERCA2a function including the activity and protein level due to AGEs accumulation. It is consistent with the results of Bidasee et al. They have shown for the first time that prolongation of cardiac relaxation rate may be attributed to diabetesinduced increase in formation of AGEs (crosslinking as well as noncrosslinking) on SERCA2a in diabetic cardiomyopathy rat. In addition, they also have found that the formation of
AGEs may decrease the amount of serine-16 and threonine17 phosphorylation of PLN [31].

However, in case of EGB761 additive, the effect of suppression was relieved. Followed by the next experiment, in order to determine which phosphorylation residue of PLN was involved in EGB761 protecting against aging-associated diastolic dysfunction in cardiomyocytes, we determined the amount of p-Ser16-PLN and p-Thr17-PLN protein. Our data showed that decreased p-Ser16-PLN protein level was observed in D-galactose-treated cardiomyocytes; while it was increased in the EGB761 group compared with the Dgalactose group $(P<0.05)$. However, the protein level of p-Thr ${ }^{17}$-PLN showed no significant changes in any of the groups compared with the control cells $(P>0.05)$. Thus, these results may imply that the activity and phosphorylation status of SERCA and PLN are involved in the pathogenesis of cardiac diastolic dysfunction. It was possible that EGB761 could upregulate SERCA2a function through improvement of the amount of Ser16 sites PLN phosphorylation, further improving the homeostasis impairment of intracellular $\mathrm{Ca}^{2+}$.

Since standard EGb761 preparation that we used mainly contains $4.2 \mathrm{mg}$ flavonoid and other unknown complex composition, our results are consistent with the reports demonstrating that flavonoid components exhibit better effects than ginkgolides and bilobalides on protection of SERCA2a function through increasing the amount of Ser16 sites PLN phosphorylation, which decline the intracellular calcium overload [32]. Abdel-Kader et al. also have confirmed that quercetin showed a similar antioxidant effect as EGb761, suggesting that ginkgo-flavone glycosides may be the major components of EGb761 and other unknown complex compositions effect on the endoplasmic reticulum, and other phenotypes tested in our paper may be multifactorial, including its antioxidant, antiglycemic and other properties $[32,33]$. In our present study, we treat EGb761 plus Dgalactose incubation cardiomyocytes that can effectively and extensively counteract this action of aging-associated diastolic dysfunction. It may be indicated that EGB761 

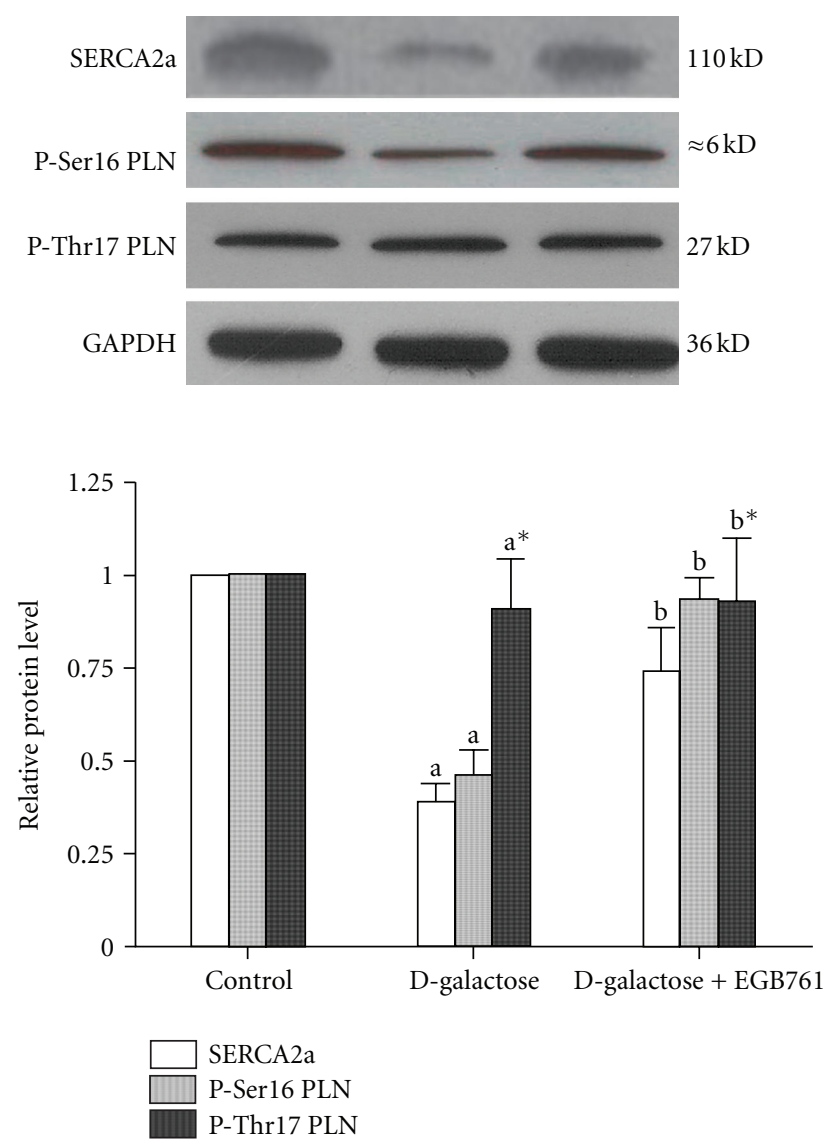

FIGURE 2: Effect of EGB761 on SERCA2a, Ser16-phosphorylated PLN (p-Ser16 PLN), Thr17-phosphorylated PLN (p-Thr17 PLN) protein levels. Representative immunoblots for SERCA2a: p-Ser16 PLN, p-Thr17 PLN, and GAPDH in samples obtained from the control (lane 1), D-galactose (lane2), D-galactose + EGB761 (lane 3). Summarized data showing SERCA2a, p-Ser16 PLN, and p-Thr17 PLN protein expression normalized. Values are expressed as mean $\pm \mathrm{SD}\left(n=4,{ }^{\mathrm{a}} \mathrm{P}<0.05\right.$ versus control group; ${ }^{\mathrm{b}} \mathrm{P}<0.05$ versus $\mathrm{D}$ galactose group).

extract may be a hypothetical preventive therapy in ageing and AGE- related disease and may potentially protect the heart from AGEs formation [11].

Taken together, due to cardiac diastolic dysfunction which is related to an elevated myocardial stiffness and the resting tension $\left(F_{\text {passive }}\right)$, it is possible that EGB761 at least partially attenuates the nonenzymatic glycation formation AGEs (crosslinking as well as noncrosslinking) on SERCA2a in cardiomyocytes on one hand, so that it mitigates myocardial stiffness. On other hand, it improves SERCA2a function through increasing the amount of Ser16 sites PLN phosphorylation, which declines the intracellular calcium overload in diastolic phase. The two hands effects of EGB761 may possibly protect against aging-associated diastolic dysfunction in cardiomyocytes. In this case, the EGB761 extract or some of its compounds may be a novel therapeutic strategy in the prevention of the cardiac alterations in diastolic dysfunction for the patients in clinical practice.

\section{Conclusions}

In summary, EGb761, as an "antiaging" herb, has been used in China for thousands of years which can treat agingassociated diseases. Our work is the first one to directly prove the anti-aging-associated diastolic dysfunction efficacy of EGB761 in cardiomyocytes in vitro. EGb761 may alleviate myocardial stiffness and the resting tension $\left(F_{\text {passive }}\right)$, thus, protecting against aging-associated diastolic dysfunction in cardiomyocytes.

\section{Authors' Contribution}

J. Liu and J. Wang contributed equally to this work.

\section{Acknowledgments}

This work was supported by grants from the National Natural Science Foundation of China (nos. 30772781 and 30900602), National Natural Science Foundation of Jiangsu Province (BK2011382), and "Sixth-Peak Talent" of Jiangsu Province (2011WSN-029) to Professor Y. Guo.

\section{References}

[1] S. Y. Li, M. Du, E. K. Dolence et al., "Aging induces cardiac diastolic dysfunction, oxidative stress, accumulation of advanced glycation endproducts and protein modification," Aging Cell, vol. 4, no. 2, pp. 57-64, 2005.

[2] G. A. Silberman, T. H. M. Fan, H. Liu et al., "Uncoupled cardiac nitric oxide synthase mediates diastolic dysfunction," Circulation, vol. 121, no. 4, pp. 519-528, 2010.

[3] J. Ren, Q. Li, S. Wu, S. Y. Li, and S. A. Babcock, "Cardiac overexpression of antioxidant catalase attenuates aging-induced cardiomyocyte relaxation dysfunction," Mechanisms of Ageing and Development, vol. 128, no. 3, pp. 276-285, 2007.

[4] X. Song, M. Bao, D. Li, and Y. M. Li, "Advanced glycation in dgalactose induced mouse aging model," Mechanisms of Ageing and Development, vol. 108, no. 3, pp. 239-251, 1999.

[5] F. Safciuc, A. Constantin, A. Manea et al., "Advanced glycation end products, oxidative stress and metalloproteinases are altered in the cerebral microvasculature during aging," Current Neurovascular Research, vol. 4, no. 4, pp. 228-234, 2007.

[6] L. Van Heerebeek, N. Hamdani, M. L. Handoko et al., "Diastolic stiffness of the failing diabetic heart: importance of fibrosis, advanced glycation end products, and myocyte resting tension," Circulation, vol. 117, no. 1, pp. 43-51, 2008.

[7] S. Dhar, D. Koul, and G. E. D’Alonzo, "Current concepts in diastolic heart failure," Journal of the American Osteopathic Association, vol. 108, no. 4, pp. 203-209, 2008.

[8] E. Picht, J. DeSantiago, S. Huke, M. A. Kaetzel, J. R. Dedman, and D. M. Bers, "CaMKII inhibition targeted to the sarcoplasmic reticulum inhibits frequency-dependent acceleration of relaxation and $\mathrm{Ca}^{2+}$ current facilitation," Journal of Molecular and Cellular Cardiology, vol. 42, no. 1, pp. 196-205, 2007.

[9] Y. C. Yeh, T. J. Liu, L. C. Wang et al., "A standardized extract of Ginkgo biloba suppresses doxorubicin-induced oxidative stress and p53-mediated mitochondrial apoptosis in rat testes," British Journal of Pharmacology, vol. 156, no. 1, pp. 4861, 2009. 
[10] J. Shen, J. Wang, B. Zhao, J. Hou, T. Gao, and W. Xin, "Effects of EGb 761 on nitric oxide and oxygen free radicals, myocardial damage and arrhythmia in ischemia-reperfusion injury in vivo," Biochimica et Biophysica Acta, vol. 1406, no. 3, pp. 228 236, 1998.

[11] J. G. Shen and D. Y. Zhou, "Efficiency of ginkgo biloba extract (EGb 761) in antioxidant protection against myocardial ischemia and reperfusion injury," Biochemistry and Molecular Biology International, vol. 35, no. 1, pp. 125-134, 1995.

[12] C. Mozet, R. Martin, K. Welt, and G. Fitz, "Cardioprotective effect of EGb 761 on myocardial ultrastructure of young and old rat heart and antioxidant status during acute hypoxia," Aging, vol. 21, no. 1, pp. 14-21, 2009.

[13] R. Schneider, K. Welt, W. Aust, H. Löster, and G. Fitzl, "Cardiac ischemia and reperfusion in spontaneously diabetic rats with and without application of EGb 761: II. Interstitium and microvasculature," Histology and Histopathology, vol. 24, no. 5, pp. 587-598, 2009.

[14] T. Liebgott, M. Miollan, Y. Berchadsky, K. Drieu, M. Culcasi, and S. Pietri, "Complementary cardioprotective effects of flavonoid metabolites and terpenoid constituents of Ginkgo biloba extract (EGb 761) during ischemia and reperfusion," Basic Research in Cardiology, vol. 95, no. 5, pp. 368-377, 2000.

[15] C. Schiborr, G. P. Eckert, J. Weissenberger et al., "Cardiac oxidative stress and inflammation are similar in SAMP8 and SAMR1 mice and unaltered by curcumin and Ginkgo biloba extract intake," Current Pharmaceutical Biotechnology, vol. 11, no. 8, pp. 861-867, 2010.

[16] D. Jezova, R. Duncko, M. Lassanova, M. Kriska, and F. Moncek, "Reduction of rise in blood pressure and cortisol release during stress by Ginkgo biloba extract (EGb 761) in healthy volunteers," Journal of Physiology and Pharmacology, vol. 53, no. 3, pp. 337-348, 2002.

[17] Y. Maejima, S. Adachi, H. Ito, K. Hirao, and M. Isobe, "Induction of premature senescence in cardiomyocytes by doxorubicin as a novel mechanism of myocardial damage," Aging Cell, vol. 7, no. 2, pp. 125-136, 2008.

[18] B. Chen, Y. Zhong, W. Peng, Y. Sun, and W. J. Kong, "Agerelated changes in the central auditory system: comparison of d-galactose-induced aging rats and naturally aging rats," Brain Research C, vol. 1344, pp. 43-53, 2010.

[19] X. L. Xu, X. J. Chen, H. Ji et al., "Astragaloside IV improved intracellular calcium handling in hypoxia-reoxygenated cardiomyocytes via the sarcoplasmic reticulum $\mathrm{Ca}^{2+}$-ATPase," Pharmacology, vol. 81, no. 4, pp. 325-332, 2008.

[20] J. V. Valencia, S. C. Weldon, D. Quinn et al., "Advanced glycation end product ligands for the receptor for advanced glycation end products: biochemical characterization and formation kinetics," Analytical Biochemistry, vol. 324, no. 1, pp. 68-78, 2004.

[21] H. M. Hsieh, W. M. Wu, and M. L. Hu, "Genistein attenuates d-galactose-induced oxidative damage through decreased reactive oxygen species and NF- $\kappa \mathrm{B}$ binding activity in neuronal PC12 cells," Life Sciences, vol. 88, no. 1-2, pp. 82-88, 2011.

[22] X. Zhang, W. Liu, X. Niu, and L. An, "Systemic administration of catalpol prevents d-galactose induced mitochondrial dysfunction in mice," Neuroscience Letters, vol. 473, no. 3, pp. 224-228, 2010.

[23] J. Pedro De Magalhães, F. Chainiaux, F. De Longueville et al., "Gene expression and regulation in $\mathrm{H}_{2} \mathrm{O}_{2}$-induced premature senescence of human foreskin fibroblasts expressing or not telomerase," Experimental Gerontology, vol. 39, no. 9, pp. 1379-1389, 2004.
[24] G. P. Dimri, X. Lee, G. Basile et al., "A biomarker that identifies senescent human cells in culture and in aging skin in vivo," Proceedings of the National Academy of Sciences of the United States of America, vol. 92, no. 20, pp. 9363-9367, 1995.

[25] D. Bernhard and G. Laufer, "The aging cardiomyocyte: a minireview," Gerontology, vol. 54, no. 1, pp. 24-31, 2008.

[26] R. Petrova, Y. Yamamoto, K. Muraki et al., "Advanced glycation endproduct-induced calcium handling impairment in mouse cardiac myocytes," Journal of Molecular and Cellular Cardiology, vol. 34, no. 10, pp. 1425-1431, 2002.

[27] D. Lagadic-Gossmann, K. J. Buckler, K. Le Prigent, and D. Feuvray, "Altered $\mathrm{Ca}^{2+}$ handling in ventricular myocytes isolated from diabetic rats," American Journal of Physiology, vol. 270, no. 5, pp. H1529-H1537, 1996.

[28] D. M. Bers, "Cardiac excitation-contraction coupling," Nature, vol. 415, no. 6868, pp. 198-205, 2002.

[29] E. Vafiadaki, V. Papalouka, D. A. Arvanitis, E. G. Kranias, and D. Sanoudou, "The role of SERCA2a/PLN complex, $\mathrm{Ca}^{2+}$ homeostasis, and anti-apoptotic proteins in determining cell fate," Pflugers Archiv European Journal of Physiology, vol. 457, no. 3, pp. 687-700, 2009.

[30] K. F. Frank, B. Bölck, E. Erdmann, and R. H. G. Schwinger, "Sarcoplasmic reticulum $\mathrm{Ca}^{2+}$-ATPase modulates cardiac contraction and relaxation," Cardiovascular Research, vol. 57, no. 1, pp. 20-27, 2003.

[31] K. R. Bidasee, Y. Zhang, C. H. Shao et al., "Diabetes increases formation of advanced glycation end products on Sarco(endo)plasmic reticulum $\mathrm{Ca}^{2+}$-ATPase," Diabetes, vol. 53, no. 2, pp. 463-473, 2004.

[32] R. Abdel-Kader, S. Hauptmann, U. Keil et al., "Stabilization of mitochondrial function by Ginkgo biloba extract (EGb 761)," Pharmacological Research, vol. 56, no. 6, pp. 493-502, 2007.

[33] J. Renugadevi and S. Milton Prabu, "Quercetin protects against oxidative stress-related renal dysfunction by cadmium in rats," Experimental and Toxicologic Pathology, vol. 62, no. 5, pp. $471-481,2010$. 


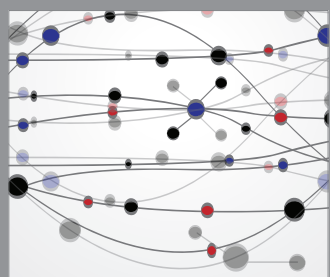

The Scientific World Journal
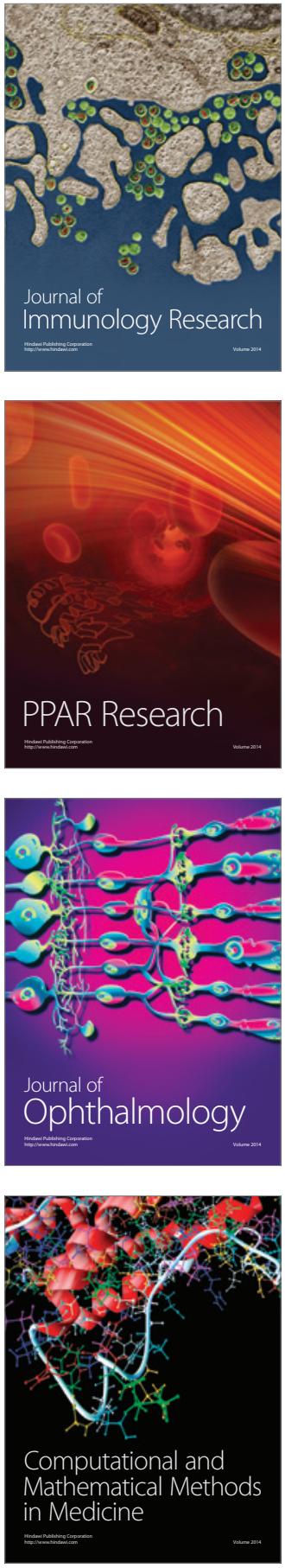

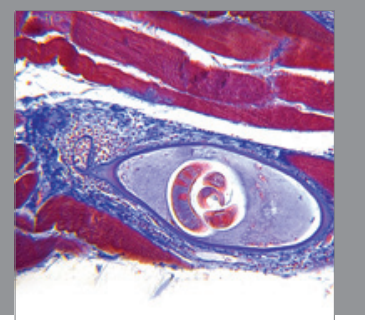

Gastroenterology

Research and Practice
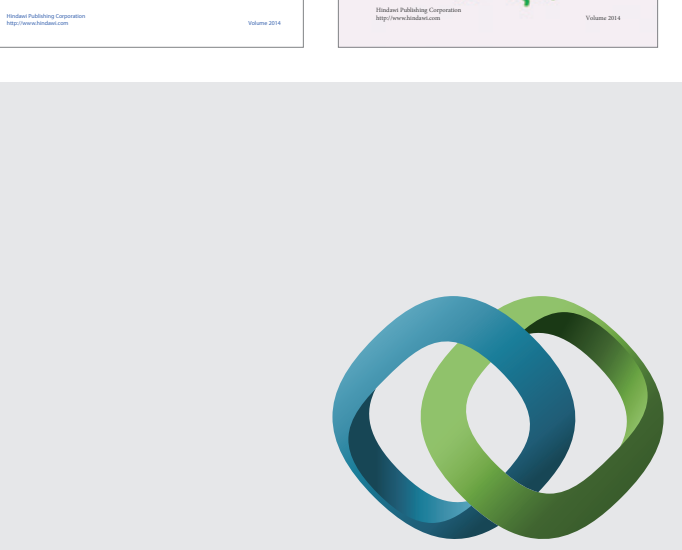

\section{Hindawi}

Submit your manuscripts at

http://www.hindawi.com
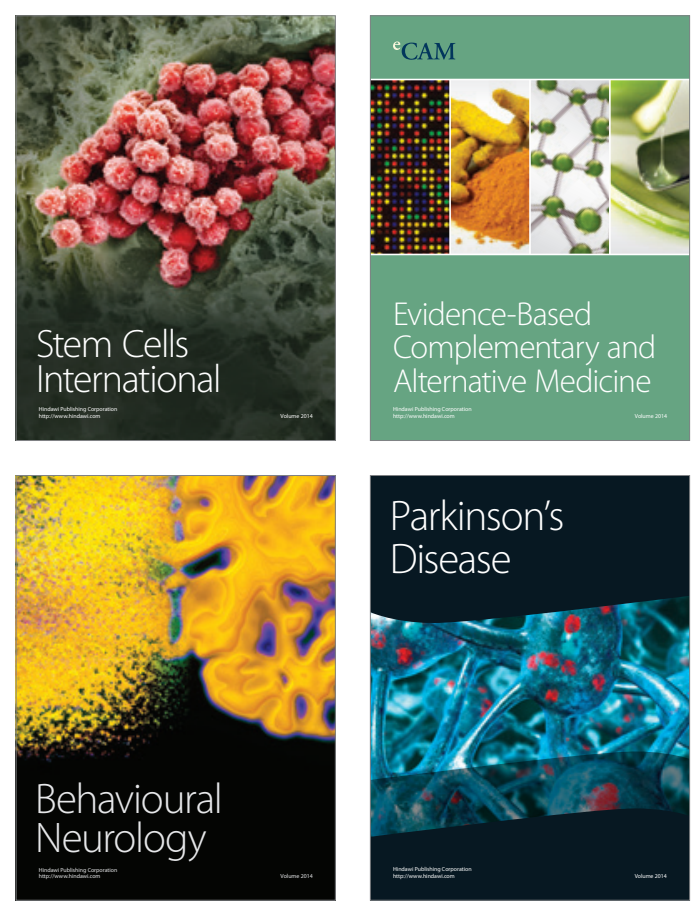

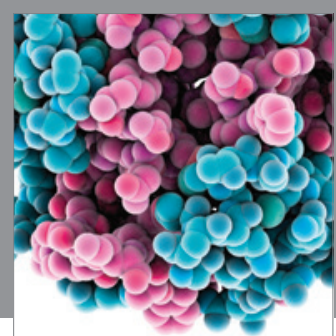

Journal of
Diabetes Research

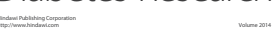

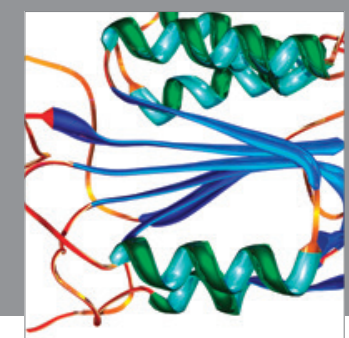

Disease Markers
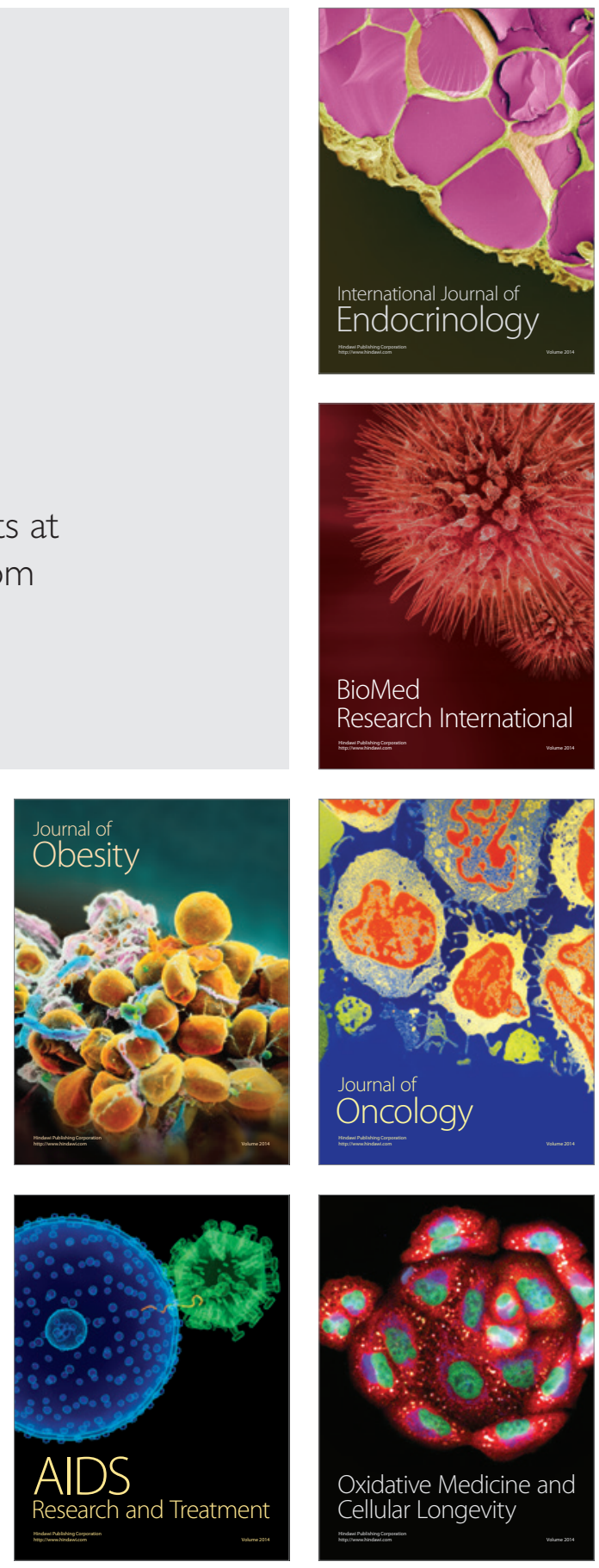